Article

\title{
Encapsulation of NEM Memory Switches for Monolithic-Three-Dimensional (M3D) CMOS-NEM Hybrid Circuits
}

\author{
Hyun Chan Jo and Woo Young Choi * (DD \\ Department of Electronics Engineering, Sogang University, Seoul 04107, Korea; jhc10337@naver.com \\ * Correspondence: wchoi@sogang.ac.kr; Tel.: +82-2-715-8467
}

Received: 30 May 2018; Accepted: 20 June 2018; Published: 23 June 2018

check for updates

\begin{abstract}
Considering the isotropic release process of nanoelectromechanical systems (NEMSs), defining the active region of NEM memory switches is one of the most challenging process technologies for the implementation of monolithic-three-dimensional (M3D) CMOS-NEM hybrid circuits. In this paper, we propose a novel encapsulation method of NEM memory switches. It uses alumina $\left(\mathrm{Al}_{2} \mathrm{O}_{3}\right)$ passivation layers which are fully compatible with the CMOS baseline process. The $\mathrm{Al}_{2} \mathrm{O}_{3}$ bottom passivation layer can protect intermetal dielectric (IMD) and metal interconnection layers from the vapor hydrogen fluoride (HF) etching process. Thus, the controllable formation of the cavity for the mechanical movement of NEM devices can be achieved without causing any damage to CMOS baseline circuits as well as metal interconnection lines. As a result, NEM memory switches can be located in any place and metal layer of an M3D CMOS-NEM hybrid chip, which makes circuit design easier and more volume efficient. The feasibility of our proposed method is verified based on experimental results.
\end{abstract}

Keywords: CMOS-NEMS; NEMS; NEM memory switch; encapsulation; M3D

\section{Introduction}

Complementary metal-oxide-semiconductor-nanoelectromechanical (CMOS-NEM) hybrid circuits have been researched intensively thanks to their unique advantages: low power consumption, high performance, low fabrication cost and high chip density [1-9]. Some pioneering experimental results of CMOS-NEM hybrid circuits have been reported [2,5]. They have NEM devices on the top of a chip or in CMOS back-end-of-line (BEOL) metal interconnection layers. For the implementation of monolithic-three-dimensional (M3D) CMOS-NEM hybrid circuits, the release process is important to form the atmospheric or vacuum environment for the mechanical motion of NEM memory switches whose operating mechanisms have already been explained elsewhere [1,2]. Generally, the release process is performed by using vapor hydrogen fluoride (HF) etching. By using the vapor HF etching, the inter-metal-dielectric (IMD) layers such as the tetraethyl orthosilicate (TEOS) layers, which surround NEM devices, can be effectively removed with high selectivity toward metal layers [10]. However, a conventional release process using vapor HF etching can cause catastrophic influences on IMD and metal interconnection layers because it is an isotropic etching process: NEM structures and adjacent metal interconnection lines collapse due to the widespread removal of IMD layers. Thus, as shown in Figure $1 \mathrm{a}, \mathrm{b}$, it is difficult to place the metal interconnection lines around NEM memory switches, which will be called the "dead zone" in this manuscript. The existence of the dead zone makes M3D CMOS-NEM hybrid circuit design difficult and volume inefficient.

To minimize the dead zone surrounding NEM devices, this manuscript proposes a novel CMOS-process-compatible encapsulation method as shown in Figure 1c. In the proposed method, 
NEM memory switches are encapsulated by alumina $\left(\mathrm{Al}_{2} \mathrm{O}_{3}\right)$ bottom/top passivation layers. The TEOS lower/upper sacrificial layers encapsulated by the $\mathrm{Al}_{2} \mathrm{O}_{3}$ bottom/top passivation layers are selectively removed by vapor HF etching while the rest of the regions are protected. Thus, the controllable formation of a cavity is feasible for the mechanical movement of NEM devices without damaging CMOS baseline circuits and metal interconnect lines. From now, this cavity will be called the "active region" of a NEM memory switch. To sum up, because our proposed encapsulation method defines the active regions of NEM devices without generating dead zones, they can be placed in any metal interconnection layers. To confirm the proposed method, prototype encapsulated NEM memory switches are implemented.

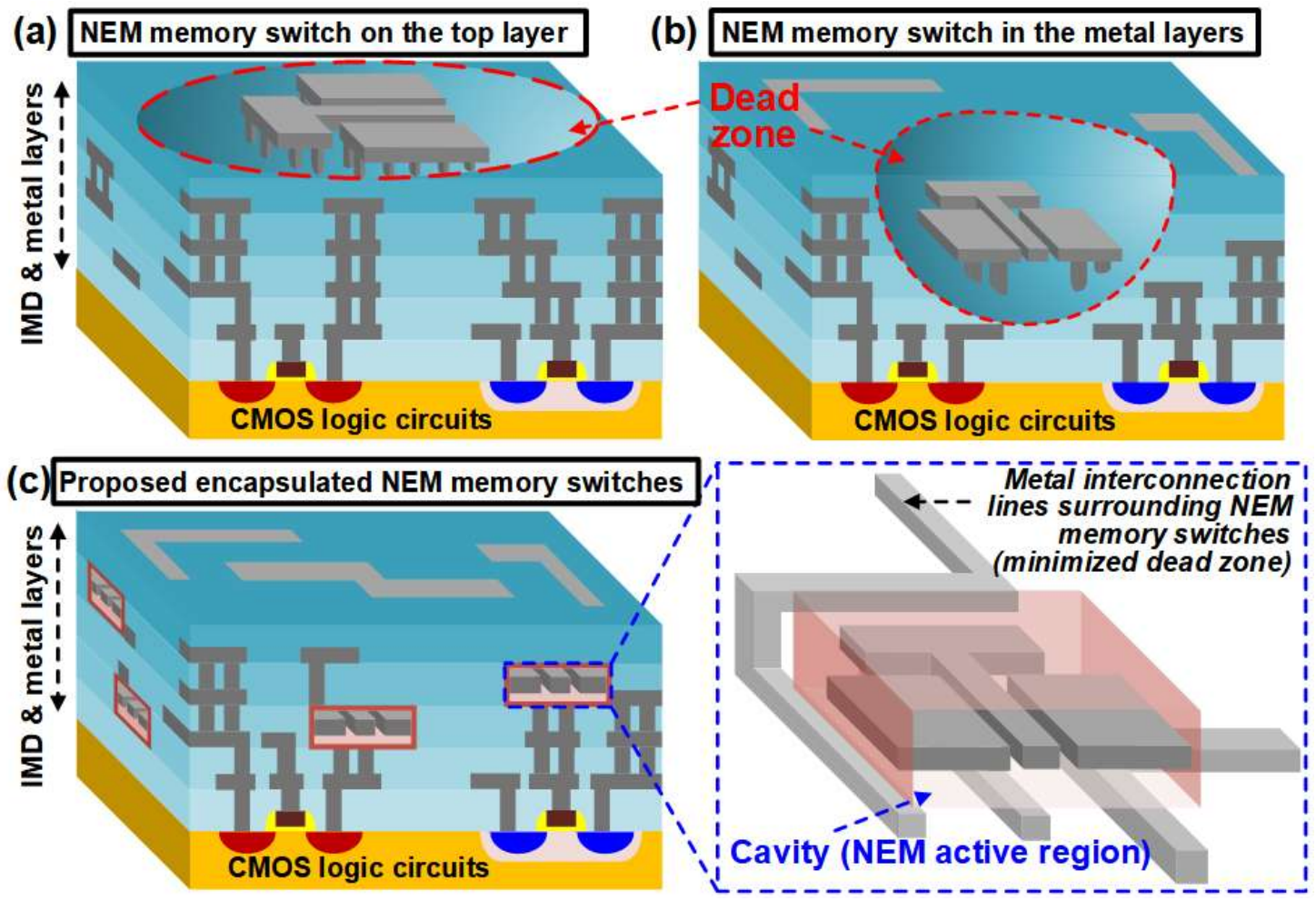

Figure 1. Conceptual views of (a) a nanoelectromechanical (NEM) memory switch only on the top layer, (b) a NEM memory switch in the CMOS back-end-of-line (BEOL) metal layers and (c) the proposed encapsulated NEM memory switches for monolithic-three-dimensional (M3D) CMOS-NEM hybrid circuits.

\section{Encapsulation Process}

Figure 2 shows the key process steps of the encapsulated nanoelectromechanical (NEM) memory switches. First, a 50-nm-thick silicon dioxide $\left(\mathrm{SiO}_{2}\right)$ layer is grown by wet oxidation. Then, a 500-nm-thick aluminum (Al) layer is sputtered and patterned by inductively coupled plasma (ICP) etching. The $\mathrm{Al}$ patterns correspond to the metal interconnect lines of CMOS baseline circuits. Third, a 500-nm-thick tetraethyl orthosilicate (TEOS) inter-metal-dielectric (IMD) layer is deposited and patterned by plasma-enhanced chemical vapor deposition (PECVD) and magnetically enhanced reactive ion etching (MERIE) processes, respectively, to define the active regions of NEM memory switches. Subsequently, a 200-nm-thick $\mathrm{Al}_{2} \mathrm{O}_{3}$ bottom passivation layer is deposited by a multisputtering process. The $\mathrm{Al}_{2} \mathrm{O}_{3}$ bottom passivation layer protects the metal interconnection lines and IMD layers from the following vapor hydrogen fluoride (HF) etch at atmospheric pressure [11-13]. Fifth, a 200-nm-thick TEOS layer is deposited as a lower sacrificial layer. Next, a 500-nm-thick Al layer is deposited and patterned to form NEM memory switches. During the patterning process, the 85-nm-wide airgap between the movable cantilever beam and selection lines is formed by a focus 
ion beam (FIB) process while the rest of the patterns are defined by a conventional stepper. Seventh, a 500-nm-thick TEOS layer is deposited and patterned as an upper sacrificial layer. It should be noted that the active regions of NEM memory switches are defined and filled by the lower and upper sacrificial layers. Eighth, a 200-nm-thick $\mathrm{Al}_{2} \mathrm{O}_{3}$ top passivation layer is deposited to encapsulate the active regions of NEM memory switches. Subsequently, small-sized etch holes are patterned on the $\mathrm{Al}_{2} \mathrm{O}_{3}$ top passivation layer by the FIB process. Tenth, the lower and upper TEOS sacrificial layers are removed through the etch holes by vapor $\mathrm{HF}$ etching at $40^{\circ} \mathrm{C}$ and $15 \mathrm{~min}$. Finally, a thick TEOS IMD layer is deposited on the $\mathrm{Al}_{2} \mathrm{O}_{3}$ top passivation layer to form the cavity surrounding NEM memory switches which acts as the active region. The encapsulated active regions are in the vacuum condition depending on TEOS deposition conditions. This encapsulation method is fully CMOS-process-compatible, which can be easily applied to the fabrication of M3D CMOS-NEM hybrid circuits.

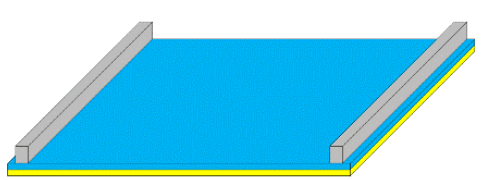

(a)

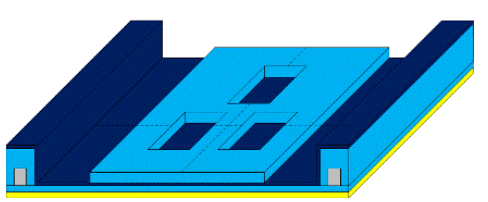

(d)

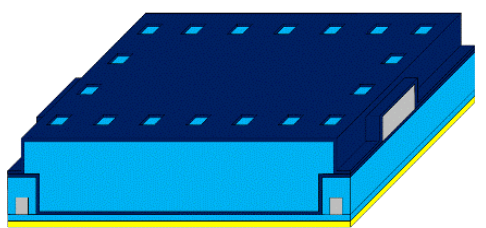

(g)

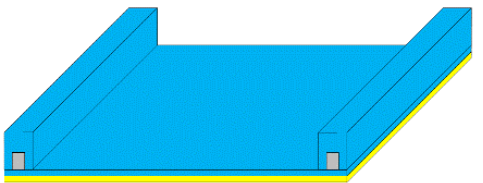

(b)

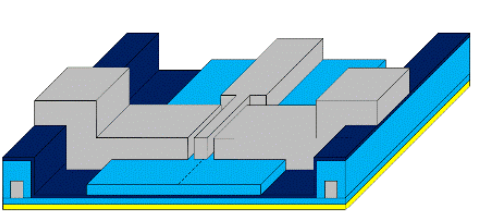

(e)

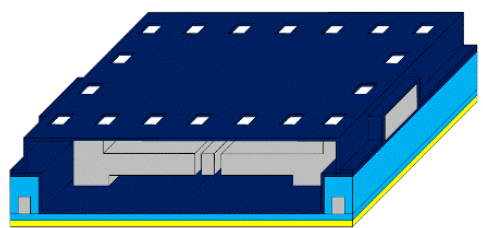

(h)

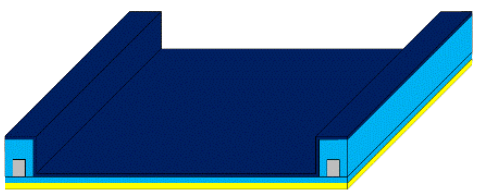

(c)

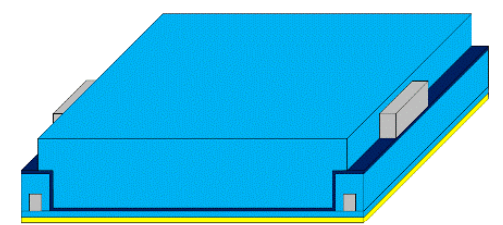

(f)

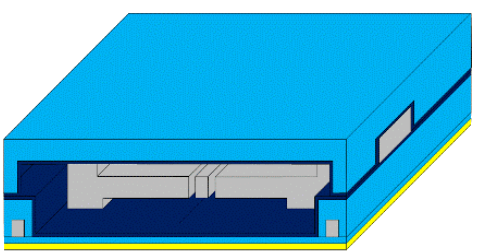

(i)

Si-substrate

TEOS

Aluminum

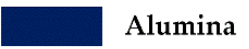

Figure 2. Key process steps of the encapsulated nanoelectromechanical (NEM) memory switches. (a) $\mathrm{Al}$ deposition and patterning for the formation of metal interconnection lines; (b) Tetraethyl orthosilicate (TEOS) deposition and patterning for inter-metal-dielectric (IMD) formation; (c) $\mathrm{Al}_{2} \mathrm{O}_{3}$ bottom passivation layer deposition; (d) Lower TEOS sacrificial layer deposition and patterning; (e) Al deposition and patterning for the formation of a NEM memory switch; (f) Upper TEOS sacrificial layer deposition and pattern; (g) $\mathrm{Al}_{2} \mathrm{O}_{3}$ top passivation layer deposition and etch hole formation; (h) Removal of the lower/upper sacrificial layers through etch holes by using vapor hydrogen fluoride (HF) etching; (i) TEOS deposition for cavity sealing.

For cavity formation, the etch holes should have the aspect ratio high enough to prevent TEOS from filling the cavity again through the etch holes. Figure 3 shows scanning electron microscopy (SEM, Thermo Fisher Scientific, Waltham, MA, USA) cross-sectional images of etch holes. In order to form the etch holes with various aspect ratios, two FIB process conditions have been adjusted: beam current and target diameter. The aspect ratio of the etch holes in Figure $3 \mathrm{a}-\mathrm{b}$ are measured to be 0.79 (beam current $=50 \mathrm{pA}$ and target diameter $=160 \mathrm{~nm}$ ) and 1.01 (beam current $=10 \mathrm{pA}$ and target diameter $=160 \mathrm{~nm}$ ), respectively. It is interesting that two different layers are observed below the $\mathrm{Al}_{2} \mathrm{O}_{3}$ top passivation layer in those two cases. The former is a thin TEOS layer which is originated from the unwanted TEOS inflow through the etch holes. It is problematic in that it prevents the motion 
of a cantilever beam of a NEM memory switch. On the contrary, the latter results from the redeposition process during the FIB sample cutting process for SEM measurement, which does not exist in the main samples [14]. Thus, to suppress TEOS inflow, the aspect ratio of the etch holes needs to be increased. If the aspect ratio is increased up to 1.14 (beam current $=10 \mathrm{pA}$ and target diameter $=80 \mathrm{~nm}$ ) as shown in Figure 3c, no unwanted TEOS inflow is observed. Only the redeposition layer originated from the FIB sample cutting process is formed under the $\mathrm{Al}_{2} \mathrm{O}_{3}$ top passivation layer.
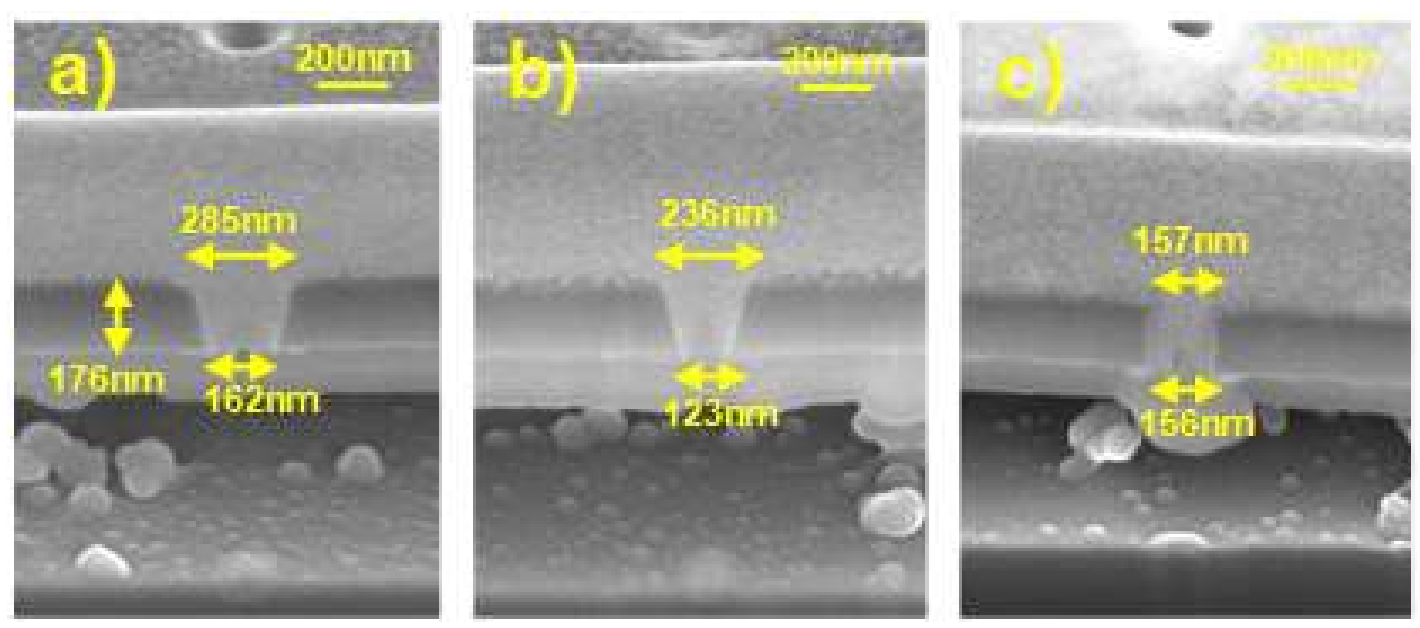

Figure 3. Cross-sectional scanning electron microscopy (SEM) images of etch holes with the variation of the beam current and target diameter of the focus ion beam (FIB) process. (a) Aspect ratio $=0.79$ when beam current is $50 \mathrm{pA}$ and target diameter is $160 \mathrm{~nm}$; (b) Aspect ratio $=1.01$ when beam current is $10 \mathrm{pA}$ and target diameter is $160 \mathrm{~nm}$. (c) Aspect ratio $=1.14$ when beam current is $10 \mathrm{pA}$ and target diameter is $80 \mathrm{~nm}$.

\section{Results and Discussion}

Figure 4 shows the SEM images of the fabricated NEM memory switch encapsulated in a cavity. Figure $4 \mathrm{a}-\mathrm{f}$ show the NEM memory switches before and after vapor hydrogen fluoride (HF) etching, respectively. The active region of the encapsulated NEM memory switch is formed well next to the metal interconnection lines, as shown in Figure 4. Figure $4 \mathrm{~b}, \mathrm{c}$ confirm that $\mathrm{Al}_{2} \mathrm{O}_{3}$ top and bottom passivation layers wrap the NEM memory switch and lower/upper tetraethyl orthosilicate (TEOS) sacrificial layers. Figure $4 \mathrm{~d}-\mathrm{f}$ show that the TEOS lower/upper sacrificial layers are successfully removed by vapor HF etching. In Figure 4e, it is confirmed that the sacrificial layers are completely removed by vapor HF without damaging the cavity regions. This forms the active region of the NEM memory switch, allowing activation between metal layers. Especially, Figure 4e shows the successful implementation of the NEM memory switch in a cavity. On the other hand, Figure $4 \mathrm{f}$ shows that the inter-metal-dielectric (IMD) layer out of the cavity is also removed by vapor HF etching, which means that the $\mathrm{Al}_{2} \mathrm{O}_{3}$ bottom passivation layer fails to protect the IMD layer from vapor $\mathrm{HF}$ etching. It is because vapor $\mathrm{HF}$ can penetrate into the $\mathrm{Al}_{2} \mathrm{O}_{3}$ layer following grain boundaries if the $\mathrm{Al}_{2} \mathrm{O}_{3}$ layer is formed by the sputtering process. Thus, in order to increase the film density of the $\mathrm{Al}_{2} \mathrm{O}_{3}$ passivation layer, an atomic layer deposition (ALD) process is used rather than a sputtering process. Figure $5 \mathrm{a}-\mathrm{d}$ show the transmission electron microscopy (TEM) images of the test sample using a 20-nm-thick ALD-deposited $\mathrm{Al}_{2} \mathrm{O}_{3}$ layer before and after 1-, 5- and 15-min vapor $\mathrm{HF}$ etching at $40{ }^{\circ} \mathrm{C}$, respectively. As predicted, it is observed that the $\mathrm{SiO}_{2} \mathrm{IMD}$ layer is completely protected by the ALD-deposited $\mathrm{Al}_{2} \mathrm{O}_{3}$ layer. 

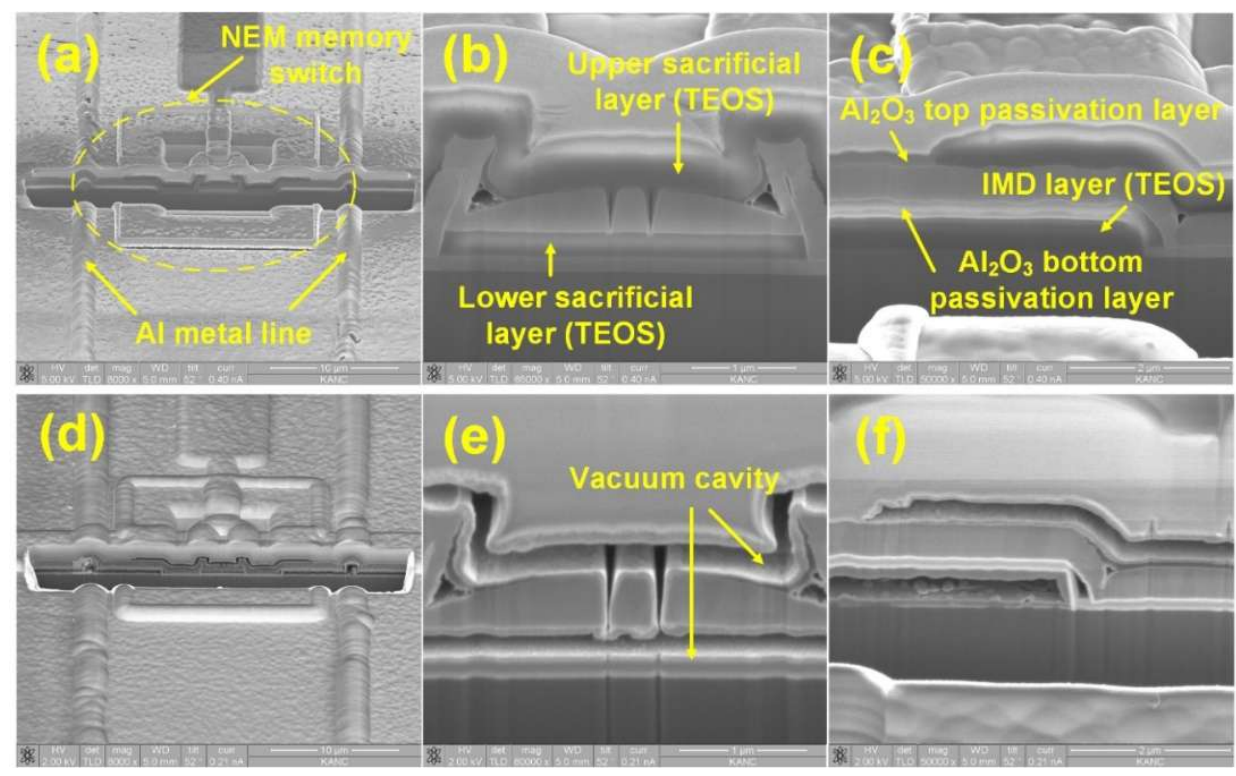

Figure 4. (a) Nanoelectromechanical (NEM) memory switch and metal interconnection lines; (b) NEM memory switch and (c) metal interconnection lines before vapor hydrogen fluoride (HF) etching; (d) NEM memory switch and metal interconnection lines; (e) NEM memory switch and (f) metal interconnection lines after vapor HF etching.
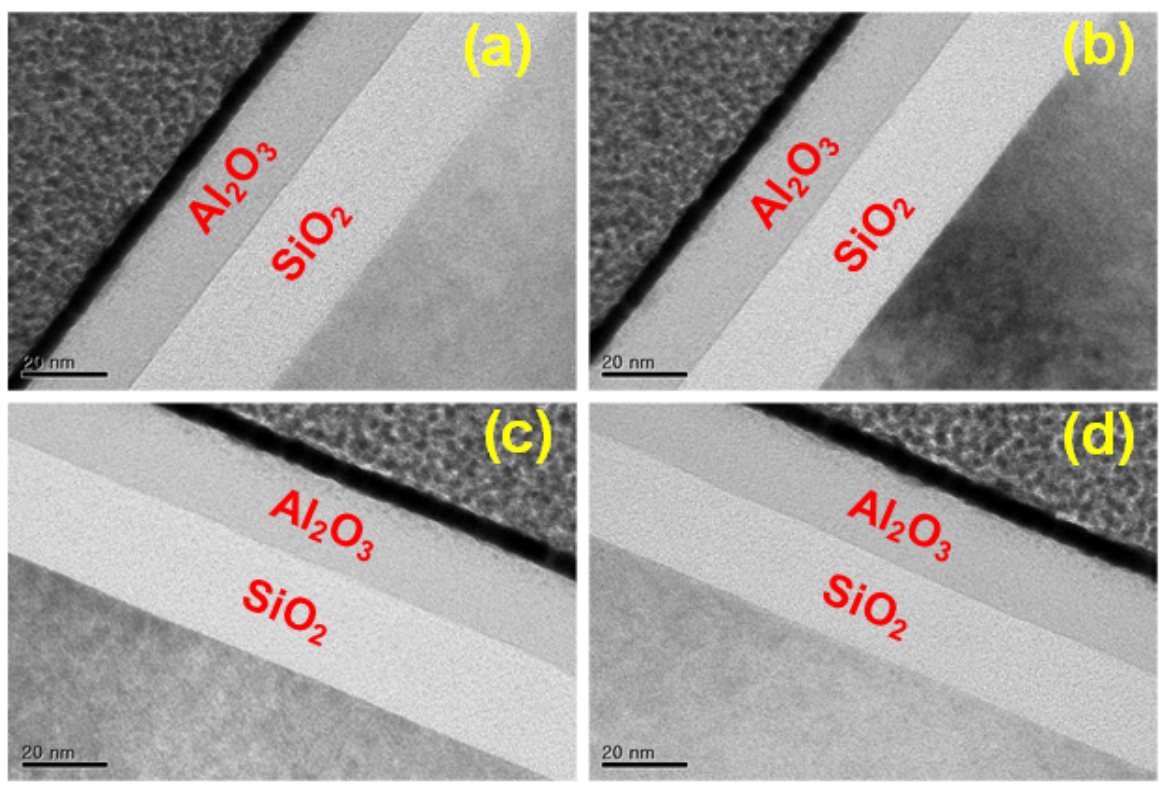

Figure 5. Transmission electron microscopy (TEM) images of an atomic layer deposition (ALD)-deposited $\mathrm{Al}_{2} \mathrm{O}_{3}$ layer (a) before and after (b) 1-min, (c) 5-min and (d) 15-min vapor hydrogen fluoride (HF) etching.

Figure 6 shows the current vs voltage curves of the fabricated NEM memory switch encapsulated in a cavity. It shows the reasonable nonvolatile switching operation between selection line $1\left(L_{1}\right)$ and selection line $2\left(L_{2}\right)$. The endurance cycle number is $\sim 11$ times due to the weak mechanical property of aluminum. In the first switching operation, the voltage difference between the movable cantilever beam and $L_{1}\left(V_{L 1}\right)$ becomes higher than the pull-in voltage $\left(V_{\text {pull-in }}\right)$, and then the movable cantilever beam is stuck onto $L_{1}$, which is called State 1 . In this case, because the adhesion force $\left(F_{a d}\right)$ is larger than the restoring spring force of the movable cantilever beam $\left(F_{r}\right)$, the movable beam remains in contact 
with $L_{1}$ even when $V_{L 1}$ is $0 V$ [15]. Thus, the nonvolatile data signal storage can be achieved. In the second switching operation, the voltage difference between the movable cantilever beam and $L_{2}\left(V_{L 2}\right)$ becomes higher than the switching voltage $\left(V_{s w i t}\right)$, and then the location of the beam tip is changed from $L_{1}$ to $L_{2}$, which is called State 2. During the measurement, maximum current level was limited to suppress microwelding effects. Poor endurance cycle number can be improved by downscaling the dimension of NEM memory switches and changing beam materials [15,16].

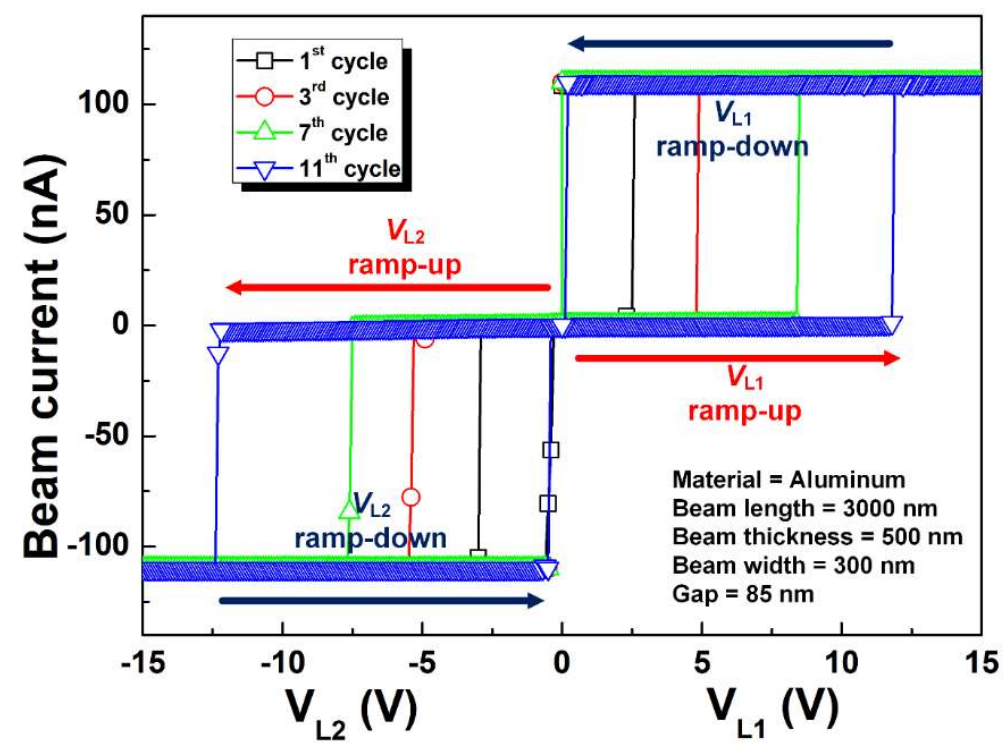

Figure 6. Current vs voltage curves of the fabricated nanoelectromechanical (NEM) memory switch encapsulated in a cavity.

\section{Conclusions}

In this work, a fabrication method to encapsulate an NEM memory switch for CMOS-NEM hybrid circuits is proposed by using a commercial CMOS process and materials. Specification of the stable encapsulated NEM memory switch is successfully confirmed based on the prototype fabrication and measurement results. By applying the proposed method confirmed in this work, the active regions of NEM memory switches can be formed without damaging CMOS baseline circuits as well as the metal interconnect lines. Because NEM memory switches can be located in any place and metal layer, the design of M3D CMOS-NEM hybrid chips can be easier and more volume efficient. It should be noted that our proposed encapsulation method can be applied to any kind of NEM device, including NEM switches, as long as they are fabricated by a CMOS backend process. For more uniform and reliable processes, a reduced-pressure vapor HF etcher can be used rather than the atmospheric-pressure vapor HF etcher used in this work. Therefore, the proposed fabrication process can lay the groundwork for commercialization of M3D CMOS-NEM hybrid circuits.

Author Contributions: W.Y.C. conceived and designed the experiments. H.C.J. performed the experiments. Both of the authors analyzed the data and wrote the paper.

Acknowledgments: This work was supported in part by the Sogang University Research Grant of 2017 (201710129.02), in part by the NRF of Korea funded by the MSIT under Grant NRF-2018R1A2A2A05019651 (Mid-Career Researcher Program), NRF-2015M3A7B7046617 (Fundamental Technology Program), NRF-2016M3A7B4909668 (Nano-Material Technology Development Program), in part by the IITP funded by the MSIT under Grant IITP-2018-0-01421 (Information Technology Research Center Program), and in part by the MOTIE/KSRC under Grant 10080575 (Future Semiconductor Device Technology Development Program).

Conflicts of Interest: The authors declare no conflict of interest. 


\section{References}

1. Choi, W.Y.; Kim, Y.J. Three-Dimensional Integration of Complementary Metal-Oxide-SemiconductorNanoelectromechanical Hybrid Reconfigurable Circuits. IEEE Electron Device Lett. 2015, 36, 887-889. [CrossRef]

2. Kwon, H.S.; Kim, S.K.; Choi, W.Y. Monolithic Three-Dimensional 65-nm CMOS-Nanoelectromechanical Reconfigurable Logic for Sub- 1.2-V Operation. IEEE Electron Device Lett. 2017, 38, 1317-1320. [CrossRef]

3. Dong, C.; Chen, C.; Mitra, S.; Chen, D. Architecture and Performance Evaluation of 3D CMOS-NEM FPGA. In Proceedings of the System Level Interconnect Prediction Workshop (SLIP), San Diego, CA, USA, 5 June 2011; pp. 1-8.

4. Chong, S.; Lee, B.G.; Parizi, K.B.; Provine, J.; Mitra, S.; Howe, R.T.; Wong, P. Integration of nanoelectromechanical (NEM) relays with silicon CMOS with functional CMOS-NEM circuit. In Proceedings of the IEEE International Electron Devices Meeting (IEDM), Washington, DC, USA, 5-7 December 2011; pp. 701-704.

5. Muñoz-Gamarra, J.; Uranga, A.; Barniol, N. CMOS-NEMS Copper Switches Monolithically Integrated Using a 65nm CMOS Technology. Micromachines 2016, 7, 30. [CrossRef]

6. Muñoz-Gamarra, J.; Alcaine, P.; Marigó, E.; Giner, J.; Uranga, A.; Esteve, J.; Barniol, N. Integration of NEMS resonators in a 65nm CMOS Technology. Microelectron. Eng. 2013, 110, 246-249. [CrossRef]

7. Riverola, M.; Vidal-Alvarez, G.; Torres, F.; Barinol, N. 3-Terminal Tungsten CMOS-NEM Relay. In Proceedings of the Ph.D. Research in Microelectronics and Electronics (PRIME), Grenoble, France, 30 June-3 July 2014.

8. Harrison, K.L.; Clary, W.A.; Provine, J.; Howe, R.T. Back-end-of-line compatible Poly-SiGe lateral nanoelectromechanical relays with multi-level interconnect. Microsyst. Technol. 2017, 23, 2125-2130. [CrossRef]

9. Riverola, M.; Uranga, A.; Torres, F.; Barniol, N. Fabrication and characterization of a hammer-shaped CMOS/BEOL-embedded nanoelectromechanical (NEM) relay. Microelectron. Eng. 2018, 192, 44-51. [CrossRef]

10. Magis, T.; Ballerand, S.; Comte, B.; Pollet, O. Deep Silicon Etch for Biology MEMS Fabrication: Review of Process Parameters Influence versus Chip Design. In Proceedings of the SPIE MOEMS-MEMS, San Francisco, CA, USA, 9 March 2013; p. 826120A.

11. Witvrouw, A.; Bois, B.D.; Moor, P.D.; Verbist, A.; Hoof, C.V.; Bender, H.; Baert, C. Comparison between Wet HF Etching and Vapor HF Etching for Sacrificial Oxide removal. In Proceedings of the SPIE Micromachining and Microfabrication, Santa Clara, CA, USA, 25 August 2000; pp. 130-141.

12. Williams, K.R.; Gupta, K.; Wasilik, M. Etch Rate for Micromachining Processing-Part II. J. Micromech. Syst. 2003, 12, 761-778. [CrossRef]

13. Bakke, T.; Schmidt, J.; Friedrichs, M.; Völker, B. Etch Stop Materials for release by vapor HF etching. In Proceedings of the MicroMechanics Europe Workshop (MME), Göteborg, Sweden, 29 January 2005; pp. 103-106.

14. Winter, D.A.M.; Mulders, J.J.L. Redeposition Characteristics of Focus Ion Beam Milling for Nanofabricaiton. J. Vac. Sci. Technol. B 2007, 25, 2215-2218. [CrossRef]

15. Choi, W.Y.; Osabe, T.; Liu, T.J.K. Nano-electro-mechanical nonvolatile memory (NEMory) cell design and scaling. IEEE Trans. Electron Devices 2008, 55, 3482-3488. [CrossRef]

16. Soon, B.W.; Ng, E.J.; Qian, Y.; Singh, N.; Tsai, M.J.; Lee, C.K. A Bi-stable Nanoelectromechanical Nonvolatile memory based on van der Waals force. Appl. Phys. Lett. 2013, 103, 053122. [CrossRef]

(C) 2018 by the authors. Licensee MDPI, Basel, Switzerland. This article is an open access article distributed under the terms and conditions of the Creative Commons Attribution (CC BY) license (http://creativecommons.org/licenses/by/4.0/). 